九州大学学術情報リポジトリ

Kyushu University Institutional Repository

\title{
A ramification theorem for the ratio of canonical forms of flat surfaces in hyperbolic three-space
}

Kawakami, Yu

Faculty of Science, Yamaguchi University

http://hdl. hand le. net/2324/20095

出版情報：Geometriae Dedicata，2013-08. Springer Netherlands バージョン :

権利関係: (C) Springer Science+Business Media Dordrecht 2013 


\section{Preprint Series}

Kyushu University

The Global COE Program

Math-for-Industry Education \& Research Hub

\section{A ramification theorem for the ratio of canonical forms of flat surfaces in hyperbolic three-space}

\section{Yu Kawakami}

MI 2011-16

( Received October 14, 2011 )

Faculty of Mathematics

Kyushu University

Fukuoka, JAPAN 


\title{
A RAMIFICATION THEOREM FOR THE RATIO OF CANONICAL FORMS OF FLAT SURFACES IN HYPERBOLIC THREE-SPACE
}

\author{
YU KAWAKAMI
}

\begin{abstract}
We provide an effective ramification theorem for the ratio of canonical forms of a weakly complete flat front in the hyperbolic three-space. Moreover we give the two applications of this theorem, the first one is to show an analogue of the Ahlfors islands theorem for it and the second one is to give a simple proof of the classification of complete nonsingular flat surfaces in the hyperbolic three-space.
\end{abstract}

\section{INTRODUCTION}

It is well-known that any complete nonsingular flat surface in the hyperbolic 3 -space $\mathbf{H}^{3}$ must be a horosphere or a hyperbolic cylinder, that is, a surface equidistance from a geodesic ([20], [21]). However if we consider flat fronts (namely, projections of Legendrian immersions) and define the notion of weakly completeness, there exist many examples and interesting global properties. For instance, more recently, Martin, Umehara and Yamada [14] showed that there exists a weakly complete bounded flat front in $\mathbf{H}^{3}$.

The ratio $\rho$ of canonical forms plays important roles in investigating the global properties of weakly complete flat fronts in $\mathbf{H}^{3}$. Indeed, Kokubu, Rossman, Saji, Umehara and Yamada [8] showed that a point $p$ is a singular point of a flat front in $\mathbf{H}^{3}$ if and only if $|\rho(p)|=1$. Moreover the author and Nakajo [6] obtained the best possible upper bound for the number of exceptional values of $\rho$ of a weakly complete flat front in $\mathbf{H}^{3}$.

The purpose of the present paper is to study the value distribution properties of the ratio of canonical forms of weakly complete flat fronts in $\mathbf{H}^{3}$. The paper is organized as follows: In Section 1, we recall some definitions and fundamental properties of flat fronts in $\mathbf{H}^{3}$, which are used throughout this paper. In Section 2, we provide a ramification theorem for the ratio of canonical forms of a weakly complete flat front in $\mathbf{H}^{3}$ (Theorem 2.2). The theorem is effective in the sense that it is sharp (see Corollary 3.4 and the comment below) and has some applications. We note that it corresponds to the defect relation in Nevanlinna theory ([7], [15], [16] and [18]). In Section 3, we give the two applications of this theorem. The first one is to show an analogue of a special case of the

2010 Mathematics Subject Classification. Primary 30D35, 53A35; Secondary 53C42.

Date: 14 October, 2011.

Key words and phrases. flat front, weakly complete, ramification theorem, the Ahlfors islands theorem, Bernstein type theorem.

The author is partially supported by the Grant-in-Aid for Young Scientists (B) No. 21740053, The Ministry of Education, Culture, Sports, Science and Technology, Japan. 
Ahlfors Islands Theorem [2, Theorem B.2] for the ratio of canonical forms of a weakly complete flat front in $\mathbf{H}^{3}$ (Corollary 3.3). We remark that Klotz and Sario [11] investigated the number of islands for the Gauss map of minimal surfaces in the Euclidean 3-space $\mathbf{R}^{3}$. The second one is to give a simple proof of the classification of complete nonsingular flat surfaces in $\mathbf{H}^{3}$ (Corollary 3.5).

Finally, the author would like to particular thank to Masatoshi Kokubu, Junjiro Noguchi, Yusuke Okuyama, Wayne Rossman, Masaaki Umehara and Kotaro Yamada for their useful advice.

\section{Preliminaries}

We briefly summarize here definitions and basic facts on flat fronts in $\mathbf{H}^{3}$ which we shall need. For more details, we refer the reader to [4], [5], [8], [9], [10], [12], [13] and [19].

Let $\mathbf{L}^{4}$ be the Lorentz-Minkowski 4 -space with inner product of signature $(-,+,+,+)$. Then the hyperbolic 3-space is given by

$$
\mathbf{H}^{3}=\left\{\left(x_{0}, x_{1}, x_{2}, x_{3}\right) \in \mathbf{L}^{4} \mid-\left(x_{0}\right)^{2}+\left(x_{1}\right)^{2}+\left(x_{2}\right)^{2}+\left(x_{3}\right)^{2}=-1, x_{0}>0\right\}
$$

with the induced metric from $\mathbf{L}^{4}$, which is a simply connected Riemannian 3-manifold with constant sectional curvature -1 . Identifying $\mathbf{L}^{4}$ with the set of $2 \times 2$ Hermitian matrices $\operatorname{Herm}(2)=\left\{X^{*}=X\right\}\left(X^{*}:={ }^{t} \bar{X}\right)$ by

$$
\left(x_{0}, x_{1}, x_{2}, x_{3}\right) \longleftrightarrow\left(\begin{array}{cc}
x_{0}+x_{3} & x_{1}+i x_{2} \\
x_{1}-i x_{2} & x_{0}-x_{3}
\end{array}\right)
$$

where $i=\sqrt{-1}$, we can write

$$
\begin{aligned}
\mathbf{H}^{3} & =\{X \in \operatorname{Herm}(2) ; \operatorname{det} X=1, \operatorname{trace} X>0\} \\
& =\left\{a a^{*} ; a \in S L(2, \mathbf{C})\right\}
\end{aligned}
$$

with the metric

$$
\langle X, Y\rangle=-\frac{1}{2} \operatorname{trace}(X \widetilde{Y}), \quad\langle X, X\rangle=-\operatorname{det}(X),
$$

where $\tilde{Y}$ is the cofactor matrix of $Y$. The complex Lie group $P S L(2, \mathbf{C}):=S L(2, \mathbf{C}) /\{ \pm$ id $\}$ acts isometrically on $\mathbf{H}^{3}$ by

$$
\mathbf{H}^{3} \ni X \longmapsto a X a^{*}
$$

where $a \in P S L(2, \mathbf{C})$.

Let $\Sigma$ be an oriented 2-manifold. A smooth map $f: \Sigma \rightarrow \mathbf{H}^{3}$ is called a front if there exists a Legendrian immersion

$$
L_{f}: \Sigma \rightarrow T_{1}^{*} \mathbf{H}^{3}
$$

into the unit cotangent bundle of $\mathbf{H}^{3}$ whose projection is $f$. Identifying $T_{1}^{*} \mathbf{H}^{3}$ with the unit tangent bundle $T_{1} \mathbf{H}^{3}$, we can write $L_{f}=(f, n)$, where $n(p)$ is a unit vector in $T_{f(p)} \mathbf{H}^{3}$ such that $\langle d f(p), n(p)\rangle=0$ for each $p \in M$. We call $n$ a unit normal vector field of the 
front $f$. A point $p \in \Sigma$ where $\operatorname{rank}(d f)_{p}<2$ is called a singularity or singular point. A point which is not singular is called regular point, where the first fundamental form is positive definite.

The parallel front $f_{t}$ of a front $f$ at distance $t$ is given by $f_{t}(p)=\operatorname{Exp}_{f(p)}(\operatorname{tn}(p))$, where "Exp" denotes the exponential map of $\mathbf{H}^{3}$. In the model for $\mathbf{H}^{3}$ as in (1.1), we can write

$$
f_{t}=(\cosh t) f+(\sinh t) n, \quad n_{t}=(\cosh t) n+(\sinh t) f,
$$

where $n_{t}$ is the unit normal vector field of $f_{t}$.

Based on the fact that any parallel surface of a flat surface is also flat at regular points, we define flat fronts as follows: A front $f: \Sigma \rightarrow \mathbf{H}^{3}$ is said to be flat if, for each $p \in M$, there exists a real number $t \in \mathbf{R}$ such that the parallel front $f_{t}$ is a flat immersion at $p$. By definition, $\left\{f_{t}\right\}$ forms a family of flat fronts. We note that an equivalent definition of flat fronts is that the Gaussian curvature of $f$ vanishes at all regular points. However, there exists a case where this definition is not suitable. For details, see [13, Remark 2.2].

We assume that $f$ is flat. Then there exists a (unique) complex structure on $\Sigma$ and a holomorphic Legendrian immersion

$$
\mathcal{E}_{f}: \widetilde{\Sigma} \rightarrow S L(2, \mathbf{C})
$$

such that $f$ and $L_{f}$ are projections of $\mathcal{E}_{f}$, where $\widetilde{\Sigma}$ is the universal covering surface of $\Sigma$. Here $\mathcal{E}_{f}$ being a holomorphic Legendrian map means that $\mathcal{E}_{f}^{-1} d \mathcal{E}_{f}$ is off-diagonal (see [4], [12], [13]). We call $\mathcal{E}_{f}$ the holomorphic Legendrian lift of $f$. The map $f$ and its unit normal vector field $n$ are

$$
f=\mathcal{E}_{f} \mathcal{E}_{f}^{*}, \quad n=\mathcal{E}_{f} e_{3} \mathcal{E}_{f}^{*}, \quad e_{3}=\left(\begin{array}{cc}
1 & 0 \\
0 & -1
\end{array}\right) .
$$

If we set

$$
\mathcal{E}_{f}^{-1} d \mathcal{E}_{f}=\left(\begin{array}{cc}
0 & \theta \\
\omega & 0
\end{array}\right),
$$

the first and second fundamental forms $d s^{2}=\langle d f, d f\rangle$ and $d h^{2}=-\langle d f, d n\rangle$ are given by

$$
\begin{aligned}
d s^{2} & =|\omega+\bar{\theta}|^{2}=Q+\bar{Q}+\left(|\omega|^{2}+|\theta|^{2}\right), \quad Q=\omega \theta \\
d h^{2} & =|\theta|^{2}-|\omega|^{2}
\end{aligned}
$$

for holomorphic 1-forms $\omega$ and $\theta$ defined on $\widetilde{\Sigma}$, with $|\omega|^{2}$ and $|\theta|^{2}$ well-defined on $\Sigma$ itself. We call $\omega$ and $\theta$ the canonical forms of $f$. The holomorphic 2-differential $Q$ appearing in the (2,0)-part of $d s^{2}$ is defined on $\Sigma$, and is called the Hopf differential of $f$. By definition, the umbilic points of $f$ coincide with the zeros of $Q$. Defining a meromorphic function on $\widetilde{\Sigma}$ by the ratio of canonical forms

$$
\rho=\frac{\theta}{\omega}
$$


then $|\rho|: \Sigma \rightarrow[0,+\infty]$ is well-defined on $\Sigma$, and $p \in \Sigma$ is a singular point if and only if $|\rho(p)|=1([8])$.

Note that the $(1,1)$-part of the first fundamental form

$$
d s_{1,1}^{2}=|\omega|^{2}+|\theta|^{2}=\left(1+|\rho|^{2}\right)|\omega|^{2}
$$

is positive definite on $\Sigma$ because it is the pull-back of the canonical Hermitian metric of $S L(2, \mathbf{C})$. Moreover $2 d s_{1,1}^{2}$ coincides with the pull-back of the Sasakian metric on $T_{1}^{*} \mathbf{H}^{3}$ by the Legendrian lift $L_{f}$ of $f$ (which is the sum of the first and third fundamental forms in this case, see [13, Section 2] for details). The complex structure on $\Sigma$ is compatible with the conformal metric $d s_{1,1}^{2}$. Note that any flat front is orientable ([9, Theorem B $]$ ). In the present paper, for each flat front $f: \Sigma \rightarrow \mathbf{H}^{3}$, we always regard $\Sigma$ as a Riemann surface with this complex structure. A flat front $f: \Sigma \rightarrow \mathbf{H}^{3}$ is said to be weakly complete if the metric $d s_{1,1}^{2}$ as in (1.11) is complete. We note that the universal cover of a weakly complete flat front is also weakly complete.

Finally, we give examples which play important roles in the following sections.

EXAmple 1.1 ([13, Example 4.1], flat fronts of revolution). Let $\bar{\Sigma}=\mathbf{C} \cup\{\infty\}$ and set

$$
\omega=-\frac{1}{c^{2}} z^{-2 /(1-\alpha)} d z, \quad \theta=\frac{c^{2} \alpha}{(1-\alpha)^{2}} z^{2 \alpha /(1-\alpha)} d z
$$

for some constants $\alpha \in \mathbf{R} \backslash\{1\}$ and $c \in \mathbf{R}$. We define $\Sigma$ by $\Sigma=\bar{\Sigma} \backslash\{0\}$ for the case where $\alpha=0$ and $\Sigma=\bar{\Sigma} \backslash\{0, \infty\}$ for the case where $\alpha \neq 0$, respectively. Then we can construct a flat front $f: \Sigma \rightarrow \mathbf{H}^{3}$ whose canonical forms are $\omega$ and $\theta$. Indeed, these data give a Legendrian immersion

$$
\mathcal{E}=\left(\begin{array}{cc}
\frac{z^{-\alpha /(1-\alpha)}}{c} & \frac{c \alpha z^{1 /(1-\alpha)}}{1-\alpha} \\
\frac{z^{-1 /(1-\alpha)}}{c} & \frac{c z^{\alpha /(1-\alpha)}}{1-\alpha}
\end{array}\right)
$$

and the corresponding flat front $f=\mathcal{E E}^{*}$ is well-defined on $\Sigma$. Moreover $f$ is weakly complete because, for each end $p \in \bar{\Sigma} \backslash \Sigma$ of $f$, it holds that

$$
\operatorname{ord}_{p} d s_{1,1}^{2}=\min \left\{\operatorname{ord}_{p}|\omega|^{2}, \operatorname{ord}_{p}|\theta|^{2}\right\} \leq-1
$$

The ratio of canonical forms of $f$ is given by

$$
\rho=\frac{\theta}{\omega}=-\frac{c^{4} \alpha}{1-\alpha} z^{2(1+\alpha) /(1-\alpha)} .
$$

Thus if $\alpha=0$ or -1 , then $\rho$ is constant. We note that $f$ is a horosphere if $\alpha=0$ or a hyperbolic cylinder if $\alpha=-1$.

Moreover we can obtain weakly complete flat fronts in $\mathbf{H}^{3}$ of Voss type ([17, Theorem 8.3], [22]). 
Proposition 1.2. Let $E$ be an arbitrary $q$ points on the Riemann sphere, where $q \leq 3$. Then there exists a weakly complete flat front in $\mathbf{H}^{3}$ whose image of the ratio of canonical forms omits precisely the set $E$.

Proof. We set $E=\left\{\alpha_{1}, \ldots, \alpha_{q-1}, \alpha_{q}\right\} \subset \mathbf{C} \cup\{\infty\}$ and $\Sigma:=\mathbf{C} \cup\{\infty\} \backslash E$. Then we may assume without loss of generality that $\alpha_{q}=\infty$. We take a holomorphic universal covering map $\xi: \widetilde{\Sigma} \rightarrow \Sigma$, where $\widetilde{\Sigma}$ is either the complex plane $\mathbf{C}$ or the unit disk. If we set

$$
\omega=\frac{d \xi}{\prod_{i=1}^{q-1}\left(\xi-\alpha_{j}\right)}, \quad \rho=\xi
$$

and use the representation (1.6), (1.7) and (1.8) on $\widetilde{\Sigma}$, we obtain a flat front in $\mathbf{H}^{3}$ whose the ratio of canonical forms omits precisely the points of $E$. Moreover it is weakly complete. Indeed, a divergent curve $\Gamma$ in $\widetilde{\Sigma}$ must tend to one of the point $w_{i}(1 \leq i \leq q)$, and we have

$$
\int_{\Gamma} d s_{1,1}=\int_{\Gamma} \sqrt{1+|\rho|^{2}}|\omega|=\int_{\Gamma} \frac{\sqrt{1+|\xi|^{2}}}{\prod_{i=1}^{q-1}\left|\xi-\alpha_{j}\right|}|d \xi|=\infty
$$

when $q \leq 3$.

\section{MAIN THEOREM}

In this section, we give an effective ramification theorem for the ratio of canonical forms of a weakly complete flat front in $\mathbf{H}^{3}$. We first recall the case where the ratio is constant.

FACT 2.1. [6, Propotion 4.4] Let $f: \Sigma \rightarrow \mathbf{H}^{3}$ be a weakly complete flat front. If the ratio of canonical forms of $f$ defined by (1.10) is constant, then $f$ is congruent to a horosphere or a hyperbolic cylinder.

The following is the main result of the present paper.

Theorem 2.2. Let $f: \Sigma \rightarrow \mathbf{H}^{3}$ be a weakly complete flat front. Let $q \in \mathbf{N}, \alpha_{1}, \ldots, \alpha_{q} \in$ $\mathbf{C} \cup\{\infty\}$ be distinct and $m_{1}, \cdots, m_{q} \in \mathbf{N}$. Suppose that

$$
\gamma=\sum_{j=1}^{q}\left(1-\frac{1}{m_{j}}\right)>3
$$

If the ratio of canonical forms $\rho: \widetilde{\Sigma} \rightarrow \mathbf{C} \cup\{\infty\}$ of $f$ satisfies the property that all $\alpha_{j}$ points of $\rho$ have multiplicity at least $m_{j}$, then $f$ must be congruent to a horosphere or a hyperbolic cylinder.

Before proceeding to the proof of Theorem 2.2, we recall two function-theoretical lemmas. For two distinct values $\alpha, \beta \in \mathbf{C} \cup\{\infty\}$, we set

$$
|\alpha, \beta|:=\frac{|\alpha-\beta|}{\sqrt{1+|\alpha|^{2}} \sqrt{1+|\beta|^{2}}}
$$


if $\alpha \neq \infty$ and $\beta \neq 0$, and $|\alpha, \infty|=|\infty, \alpha|:=1 / \sqrt{1+|\alpha|^{2}}$. Note that, if we take $v_{1}$, $v_{2} \in \mathbf{S}^{2}$ with $\alpha=\varpi\left(v_{1}\right)$ and $\beta=\varpi\left(v_{2}\right)$, we have that $|\alpha, \beta|$ is a half of the chordal distance between $v_{1}$ and $v_{2}$, where $\varpi$ denotes the stereographic projection of the 2 -sphere $\mathbf{S}^{2}$ onto $\mathbf{C} \cup\{\infty\}$.

LEMma 2.3 ([3, Corollary 1.4.15]). Let $\rho$ be a nonconstant meromorphic function on $\triangle_{R}=\{z \in \mathbf{C} ;|z|<R\}(0<R \leq \infty)$. Let $q \in \mathbf{N}, \alpha_{1}, \ldots, \alpha_{q} \in \mathbf{C} \cup\{\infty\}$ be distinct and $m_{1}, \cdots, m_{q} \in \mathbf{N}$. Suppose that

$$
\gamma=\sum_{j=1}^{q}\left(1-\frac{1}{m_{j}}\right)>2 .
$$

If $\rho$ satisfies the property that all $\alpha_{j}$-points of $\rho$ have multiplicity at least $m_{j}$, then, for arbitrary constants $\eta \geq 0$ and $\delta>0$ with $\gamma-2>\gamma(\eta+\delta)$, it holds that

$$
\frac{\left|\rho^{\prime}\right|}{1+|\rho|^{2}} \frac{1}{\left(\prod_{j=1}^{q}\left|\rho, \alpha_{j}\right|^{1-1 / m_{j}}\right)^{1-\eta-\delta}} \leq C \frac{R}{R^{2}-|z|^{2}},
$$

where $C$ is some constant depending only on $\gamma, \eta, \delta$, and $L:=\min _{i<j}\left|\alpha_{i}, \alpha_{j}\right|$.

Lemma 2.4 ([3, Corollary 1.6.7]). Let $d \sigma^{2}$ be a conformal flat metric on an open Riemann surface $\Sigma$. Then, for each point $p \in \Sigma$, there exists a local diffeomorphism $\Phi$ of a disk $\Delta_{R}=\{z \in \mathbf{C} ;|z|<R\} \quad(0<R \leq+\infty)$ onto an open neighborhood of $p$ with $\Phi(0)=p$ such that $\Phi$ is a local isometry, that is, the pull-back $\Phi^{*}\left(d \sigma^{2}\right)$ is equal to the standard Euclidean metric $d s_{E u c}^{2}$ on $\Delta_{R}$ and, for a point $a_{0}$ with $\left|a_{0}\right|=1$, the $\Phi$-image $\Gamma_{a_{0}}$ of the curve $L_{a_{0}}=\left\{w:=a_{0} s ; 0<s<R\right\}$ is divergent in $\Sigma$.

Proof of Theorem 2.2. This is proved by contradiction. Suppose that $\rho$ is nonconstant. For our purpose, we may assume $\alpha_{q}=\infty$ and that $\widetilde{\Sigma}$ is biholomorphic to the unit disk because Theorem 2.2 is obvious in the case where $\widetilde{\Sigma}=\mathbf{C}$ by Nevanlinna theory [15, Section 3 in Chapter X]. We choose some $\delta$ such that $\gamma-3>2 \gamma \delta>0$ and set

$$
\eta:=\frac{\gamma-3-2 \gamma \delta}{\gamma}, \quad \lambda=\frac{1}{1+\gamma \delta} .
$$

Then if we choose a sufficiently small positive number $\delta$ depending only on $\gamma$, for the constant $\varepsilon_{0}=(\gamma-3) / 2 \gamma$ we have

$$
0<\lambda<1, \quad \frac{\varepsilon_{0} \lambda}{1-\lambda}\left(=\frac{\gamma-3}{2 \gamma^{2} \delta}\right)>1 .
$$

Now we define a new metric

$$
d \sigma^{2}=\left|h_{z}\right|^{2 /(1-\lambda)}\left(\frac{1}{\left|\rho_{z}^{\prime}\right|} \prod_{j=1}^{q-1}\left(\frac{\left|\rho-\alpha_{j}\right|}{\sqrt{1+\left|\alpha_{j}\right|}}\right)^{\eta_{j}(1-\eta-\delta)}\right)^{2 \lambda /(1-\lambda)}|d z|^{2}
$$

on the set $\widetilde{\Sigma}^{\prime}=\left\{z \in \widetilde{\Sigma} ; \rho_{z}^{\prime}(z) \neq 0\right.$ and $\rho(z)=a_{j}$ for all $\left.j\right\}$ where $\omega=h_{z} d z, \rho_{z}^{\prime}=d \rho / d z$ and $\eta_{j}=1-1 / m_{j}$. Take a point $p \in \widetilde{\Sigma}^{\prime}$. Since the metric $d \sigma^{2}$ is flat on $\widetilde{\Sigma}^{\prime}$, by Lemma 2.4 , 
there exists a local isometry $\Phi$ satisfying $\Phi(0)=p$ from a disk $\triangle_{R}=\{z \in \mathbf{C} ;|z|<R\}$ $(0<R \leq+\infty)$ with the standard metric $d s_{E u c}^{2}$ onto an open neighborhood of $p$ in $\widetilde{\Sigma}^{\prime}$ with the metric $d \sigma^{2}$ such that, for a point $a_{0}$ with $\left|a_{0}\right|=1$, the $\Phi$-image $\Gamma_{a_{0}}$ of the curve $L_{a_{0}}=\left\{w:=a_{0} s ; 0<s<R\right\}$ is divergent in $\widetilde{\Sigma}^{\prime}$. For brevity, we denote the function $\rho \circ \Phi$ on $\triangle_{R}$ by $\rho$ in the followings. By Lemma 2.3, we get

$$
R \leq C \frac{1+|\rho(0)|^{2}}{\left|\rho_{z}^{\prime}(0)\right|} \prod_{j=1}^{q}\left|\rho(0), \alpha_{j}\right|^{\eta_{j}(1-\eta-\delta)}<+\infty .
$$

Hence

$$
L_{d \sigma}\left(\Gamma_{a_{0}}\right)=\int_{\Gamma_{a_{0}}} d \sigma=R<+\infty
$$

where $L_{d \sigma}\left(\Gamma_{a_{0}}\right)$ denotes the length of $\Gamma_{a_{0}}$ with respect to the metric $d \sigma^{2}$.

Now we prove that $\Gamma_{a_{0}}$ is divergent in $\widetilde{\Sigma}$. If not, then $\Gamma_{a_{0}}$ must tend to a point $p_{0} \in \widetilde{\Sigma} \backslash \widetilde{\Sigma}^{\prime}$ where $\rho_{z}^{\prime}\left(p_{0}\right)=0$ or $\rho\left(p_{0}\right)=\alpha_{j}$ for some $j$ because $\Gamma_{a_{0}}$ is divergent in $\widetilde{\Sigma}^{\prime}$ and $L_{d \sigma}\left(\Gamma_{a_{0}}\right)<$ $+\infty$. Taking a local complex coordinate $\zeta$ in a neighborhood of $p_{0}$ with $\zeta\left(p_{0}\right)=0$, we can write the metric $d \sigma^{2}$ as

$$
d \sigma^{2}=|\zeta|^{2 k \lambda /(1-\lambda)} v|d z|^{2}
$$

with some positive smooth function $v$ and some real number $k$. If $\rho-\alpha_{j}$ has a zero of order $m\left(\geq m_{j} \geq 2\right)$ at $p_{0}$ for some $j \leq q-1$, then $\rho_{z}^{\prime}$ has a zero of order $m-1$ at $p_{0}$ and $h_{z}\left(p_{0}\right) \neq 0$. Then we have

$$
\begin{aligned}
k & =-(m-1)+m\left(1-\frac{1}{m_{j}}\right)(1-\eta-\delta) \\
& =1-\frac{m}{m_{j}}-\frac{m}{m_{j}}\left(m_{j}-1\right)(\eta+\delta) \\
& \leq-(\eta+\delta) \leq-\varepsilon_{0} .
\end{aligned}
$$

For the case where $\rho$ has a pole of order $m\left(\geq m_{q}\right), \rho_{z}^{\prime}$ has a pole of order $m+1, h_{z}$ has a zero of order $m$ at $p_{0}$ and each component $\rho-\alpha_{j}$ in the right side of (2.5) has a pole of order $m$ at $p_{0}$. Using the identity $\eta_{1}+\cdots+\eta_{q-1}=\gamma-\eta_{q}$ and (2.4), we get

$$
\begin{aligned}
k & =\frac{m}{\lambda}+(m+1)-m\left(\gamma-\eta_{q}\right)(1-\eta-\delta) \\
& =m \eta_{q}(1-\eta-\delta)-(m-1) \leq-\varepsilon_{0} .
\end{aligned}
$$

Moreover, for the case where $\rho_{z}^{\prime}\left(p_{0}\right)=0$ and $\rho\left(p_{0}\right) \neq \alpha_{j}$ for all $j$, we see $k \leq-1$. Thus, in any case, $k \lambda /(1-\lambda) \leq-1$ by $(2.4)$ and there exists a positive constant $C^{\prime}$ such that

$$
d \sigma \geq C^{\prime} \frac{|d \zeta|}{|\zeta|}
$$

in a neighborhood of $p_{0}$. Hence we have

$$
R=\int_{\Gamma_{a_{0}}} d \sigma \geq C^{\prime} \int_{\Gamma_{a_{0}}} \frac{|d \zeta|}{|\zeta|}=+\infty
$$

which contradicts (2.6). 
On the other hand, since $d \sigma^{2}=|d z|^{2}$, we obtain by $(2.5)$

$$
\left|h_{z}\right|=\left(\left|\rho_{z}^{\prime}\right| \prod_{j=1}^{q-1}\left(\frac{\sqrt{1+\left|\alpha_{j}\right|^{2}}}{\left|\rho-\alpha_{j}\right|}\right)^{\eta_{j}(1-\eta-\delta)}\right)^{\lambda}
$$

By Lemma 2.3, we have

$$
\begin{aligned}
\Phi^{*} d s_{1,1} & =\left|h_{z}\right| \sqrt{1+|\rho|^{2}}|d z| \\
& =\left(\left|\rho_{z}\right|\left(1+|\rho|^{2}\right)^{1 / 2 \lambda} \prod_{j=1}^{q-1}\left(\frac{\sqrt{1+\left|\alpha_{j}\right|^{2}}}{\left|\rho-\alpha_{j}\right|}\right)^{\eta_{j}(1-\eta-\delta)}\right)^{\lambda}|d z| \\
& =\left(\frac{\left|\rho_{z}^{\prime}\right|}{1+|\rho|^{2}} \frac{1}{\prod_{j=1}^{q}\left|\rho, \alpha_{j}\right|^{\eta_{j}(1-\eta-\delta)}}\right)^{\lambda}|d z| \\
& \leq C^{\lambda}\left(\frac{R}{R^{2}-|z|^{2}}\right)^{\lambda}|d z|
\end{aligned}
$$

Thus if we denote the distance $d(p)$ from a point $p \in \widetilde{\Sigma}$ to the boundary of $\widetilde{\Sigma}$ as the greatest lower bound of the lengths with respect to the metric $d s_{1,1}^{2}$ of all divergent paths in $\widetilde{\Sigma}$, then we have

$$
d(p) \leq \int_{\Gamma_{a_{0}}} d s_{1,1}=\int_{L_{a_{0}}} \Phi^{*} d s_{1,1}=C^{\lambda} \int_{L_{a_{0}}}\left(\frac{R}{R^{2}-|z|^{2}}\right)^{\lambda}|d z| \leq C^{\lambda} \frac{R^{1-\lambda}}{1-\lambda}<+\infty
$$

because $0<\lambda<1$. However it contradicts the assumption that $d s_{1,1}^{2}$ is complete.

\section{Applications}

This section is devoted to prove two applications of the main theorem.

3.1. The Ahlfors Islands Theorem. We recall the definition of an island of a meromorphic function on a Riemann surface.

Definition 3.1. Let $\Sigma$ be a Riemann surface and $f: \Sigma \rightarrow \mathbf{C} \cup\{\infty\}$ a meromorphic function. Let $V \subset \mathbf{C} \cup\{\infty\}$ be a Jordan domain. A simply-connected component $U$ of $f^{-1}(V)$ with $\bar{U} \subset \Sigma$ is called an island of $f$ over $V$. Note that $\left.f\right|_{U}: U \rightarrow V$ is a proper map. The degree of this map is called the multiplicity of the island $U$. An island of multiplicity one is called a simple island.

Since the ratio $\rho$ of canonical forms of a flat front $f: \Sigma \rightarrow \mathbf{H}^{3}$ is a meromorphic function on $\widetilde{\Sigma}$, we can consider an island of $\rho$. When all islands of $\rho$ are small disks, we get the following result by applying Theorem 2.2.

Corollary 3.2. Let $f: \Sigma \rightarrow \mathbf{H}^{3}$ be a weakly complete flat front. Let $q \in \mathbf{N}, \alpha_{1}, \ldots, \alpha_{q} \in$ $\mathbf{C}$ be distinct, $D\left(\alpha_{j}, \varepsilon\right):=\left\{z \in \mathbf{C}:\left|z-\alpha_{j}\right|<\varepsilon\right\}(1 \leq j \leq q)$ be pairwise disjoint and 
$m_{1}, \ldots, m_{q} \in \mathbf{N}$. Suppose that

$$
\sum_{j=1}^{q}\left(1-\frac{1}{m_{j}}\right)>3 .
$$

Then there exists $\varepsilon>0$ such that if the ratio of canonical forms of $f$ has no island of multiplicity less than $m_{j}$ over $D\left(\alpha_{j}, \varepsilon\right)$ for all $j \in\{1, \ldots, q\}$ then $f$ must be congruent to a horosphere or a hyperbolic cylinder.

Proof. If such an $\varepsilon$ does not exist, for any $\varepsilon$, we can find a weakly complete flat front whose the ratio of canonical forms $\rho$ is nonconstant and has no island of multiplicity less than $m_{j}$ over $D\left(\alpha_{j}, \varepsilon\right)$. However this implies that all $\alpha_{j}$-points of $\rho$ have multiplicity at least $m_{j}$, contradicting Theorem 2.2 .

The important special case of Corollary 3.2 is the case where $q=7$ and $m_{j}=2$ for all $j$. This corresponds to a weak version of the so-called Five Islands Theorem in the Ahlfors theory of covering surfaces ([1], [15, Chapter XIII]).

Corollary 3.3. Let $f: \Sigma \rightarrow \mathbf{H}^{3}$ be a weakly complete flat front. Let $\alpha_{1}, \ldots, \alpha_{7} \in \mathbf{C}$ be distinct and $D\left(\alpha_{j}, \varepsilon\right):=\left\{z \in \mathbf{C}:\left|z-\alpha_{j}\right|<\varepsilon\right\}(1 \leq j \leq 7)$. Then there exists $\varepsilon>0$ such that if the ratio of canonical forms $\rho$ of $f$ has no simple island of over any of the small disks $D\left(\alpha_{j}, \varepsilon\right)$ then $f$ must be congruent to a horosphere or a hyperbolic cylinder.

3.2. The classification of complete flat surfaces in $\mathbf{H}^{3}$. As another application of Theorem 2.2, we obtain the best possible upper bound for the number of exceptional values of the ratio of canonical forms of a weakly complete flat front in $\mathbf{H}^{3}$.

Corollary 3.4 ([6, Theorem 4.5]). Let $f$ be a weakly complete flat front in $\mathbf{H}^{3}$. If the ratio of canonical forms $\rho$ of $f$ omits more than three values, then $f$ must be congruent to a horosphere or a hyperbolic cylinder.

Proof. In Theorem 2.2, if $\rho$ does not take a value $\alpha_{j}$, then we may set $m_{j}=\infty$ in (2.1), and if $\rho$ omits all values $\alpha_{j}(1 \leq j \leq q),(2.1)$ means $q>3$, which is the case of this result.

The number "three" is sharp because there exist examples in Proposition 1.2. As an application of this corollary, we give a simple proof of the classification of complete nonsingular flat surfaces in $\mathbf{H}^{3}$.

Corollary 3.5 ([20], [21]). Any complete flat surface in $\mathbf{H}^{3}$ must be congruent to a horosphere or a hyperbolic cylinder.

Proof. Because a flat surface has no singularities, the complement of the image of $\rho$ contains at least the set $\{|\rho|=1\} \subset \mathbf{C} \cup\{\infty\}$. On the other hand, we have

$$
d s^{2}=|\omega+\bar{\theta}|^{2} \leq|\omega|^{2}+|\theta|^{2} .
$$


Thus if a flat surface in $\mathbf{H}^{3}$ is complete, then it is also weakly complete. Therefore, by Corollary 3.4, it must be congruent to a horosphere or a hyperbolic cylinder.

\section{REFERENCES}

[1] L. V. Ahlfors, Zur Theorie der Überlagerungsflächen, Acta Math. 65 (1935), 157-194, and Collected Papers Vol. I, pp. 163-173.

[2] W. Bergweiler, The role of the Ahlfors five islands theorem in complex dynamics, Conform. Geom. Dyn 4 (2000), 22-34.

[3] H. Fujimoto, Value distribution theory of the Gauss map of minimal surfaces in $\mathbf{R}^{m}$, Vieweg, Braunschweig/Wiesbaden, 1993.

[4] J. A. Gálvez, A. Martínez and F. Milán, Flat surfaces in hyperbolic 3-space, Math. Ann. 316 (2000), 419-435.

[5] Y. Kawakami, Value distribution of the hyperbolic Gauss maps for flat fronts in hyperbolic threespace, to appear in Houston Journal of Mathematics, arXiv:0908.1307.

[6] Y. Kawakami and D. Nakajo, Value distribution of the Gauss map of improper affine spheres, to appear in Journal of the Mathematical Society of Japan, arXiv:1004.1484.

[7] R. Kobayashi, Toward Nevanlinna theory as a geometric model of Diophantine approximation, Sugaku Expositions 16 (2003), 39-79.

[8] M. Kokubu, W. Rossman, K. Saji, M. Umehara and K. Yamada, Singularities of flat fronts in hyperbolic space, Pacific J. Math. 221 (2005), 303-351.

[9] M. Kokubu, W. Rossman, M. Umehara and K. Yamada, Flat fronts in hyperbolic 3-space and their caustics, J. Math. Soc. Japan 59 (2007), 265-299.

[10] M. Kokubu, W. Rossman, M. Umehara and K. Yamada, Asymptotic behavior of flat surfaces in hyperbolic 3-space, J. Math. Soc. Japan 61 (2009), 799-852.

[11] T. Klotz and L. Sario, Gaussian mapping of arbitrary minimal surfaces, J. Analyse Math. 17 (1966), 209-217.

[12] M. Kokubu, M. Umehara and K. Yamada, An elementary proof of Small's formula for null curves in $\operatorname{PSL}(2, \mathbf{C})$ and an analogue for Legendrian curves in PSL(2, C), Osaka J. Math. 40 (2003), 697-715.

[13] M. Kokubu, M. Umehara and K. Yamada, Flat fronts in hyperbolic 3-space, Pacific J. Math. 216 (2004), 149-175.

[14] F. Martín, M. Umehara and K. Yamada, Calabi-Yau problem for Legendrian curves in $\mathbb{C}^{3}$ and applications, preprint, arXiv:1107.0106.

[15] R. Nevanlinna, Analytic Function, Translated from the second German edition by Phillip Emig. Die Grundlehren der mathematischen Wissenschaften, Springer, New York, 1970.

[16] J. Noguchi and T. Ochiai, Geometric Function Theory in Several Complex Variables, Transl. Math. Monog. 80, Amer. Math. Soc., Providence, RI, 1990.

[17] R. Osserman, A survey of minimal surfaces, Second edition. Dover Publications, Inc., New York, 1986.

[18] M. Ru, Nevanlinna theory and its relation to Diophantine approximation, World Sci. Publ. Co. Inc., River Edge, NJ, 2001.

[19] K. Saji, M. Umehara and K. Yamada, The geometry of fronts, Ann. of Math. 169 (2009), 491-529.

[20] S. Sasaki, On complete flat surfaces in hyperbolic 3-space, Kōdai Math Sem. Rep. 25 (1973), 449-457.

[21] Y. A. Volkov and S. M. Vladimirova, Isometric immersions of the Euclidean plane in Lobačevskii space (Russian), Mat. Zametki 10 (1971), 327-332.

[22] K. Voss, Uber vollstandige minimalflachen, L'Enseignement Math., 10 (1964), 316-317. 
Graduate School of Science and Engineering,

YAMAGUCHI UNIVERSITY,

YAMAGUCHI, 753-8512, JAPAN

E-mail address: y-kwkami@yamaguchi-u.ac.jp 


\section{List of MI Preprint Series, Kyushu University}

The Global COE Program

Math-for-Industry Education \& Research Hub

MI

MI2008-1 Takahiro ITO, Shuichi INOKUCHI \& Yoshihiro MIZOGUCHI

Abstract collision systems simulated by cellular automata

MI2008-2 Eiji ONODERA

The intial value problem for a third-order dispersive flow into compact almost

Hermitian manifolds

MI2008-3 Hiroaki KIDO

On isosceles sets in the 4-dimensional Euclidean space

MI2008-4 Hirofumi NOTSU

Numerical computations of cavity flow problems by a pressure stabilized characteristiccurve finite element scheme

MI2008-5 Yoshiyasu OZEKI

Torsion points of abelian varieties with values in nfinite extensions over a padic field

MI2008-6 Yoshiyuki TOMIYAMA

Lifting Galois representations over arbitrary number fields

MI2008-7 Takehiro HIROTSU \& Setsuo TANIGUCHI

The random walk model revisited

MI2008-8 Silvia GANDY, Masaaki KANNO, Hirokazu ANAI \& Kazuhiro YOKOYAMA Optimizing a particular real root of a polynomial by a special cylindrical algebraic decomposition

MI2008-9 Kazufumi KIMOTO, Sho MATSUMOTO \& Masato WAKAYAMA

Alpha-determinant cyclic modules and Jacobi polynomials 
MI2008-10 Sangyeol LEE \& Hiroki MASUDA

Jarque-Bera Normality Test for the Driving Lévy Process of a Discretely Observed Univariate SDE

MI2008-11 Hiroyuki CHIHARA \& Eiji ONODERA

A third order dispersive flow for closed curves into almost Hermitian manifolds

MI2008-12 Takehiko KINOSHITA, Kouji HASHIMOTO and Mitsuhiro T. NAKAO

On the $L^{2}$ a priori error estimates to the finite element solution of elliptic problems with singular adjoint operator

MI2008-13 Jacques FARAUT and Masato WAKAYAMA

Hermitian symmetric spaces of tube type and multivariate Meixner-Pollaczek polynomials

MI2008-14 Takashi NAKAMURA

Riemann zeta-values, Euler polynomials and the best constant of Sobolev inequality

MI2008-15 Takashi NAKAMURA

Some topics related to Hurwitz-Lerch zeta functions

MI2009-1 Yasuhide FUKUMOTO

Global time evolution of viscous vortex rings

MI2009-2 Hidetoshi MATSUI \& Sadanori KONISHI

Regularized functional regression modeling for functional response and predictors

MI2009-3 Hidetoshi MATSUI \& Sadanori KONISHI

Variable selection for functional regression model via the $L_{1}$ regularization

MI2009-4 Shuichi KAWANO \& Sadanori KONISHI

Nonlinear logistic discrimination via regularized Gaussian basis expansions

MI2009-5 Toshiro HIRANOUCHI \& Yuichiro TAGUCHII

Flat modules and Groebner bases over truncated discrete valuation rings 
MI2009-6 Kenji KAJIWARA \& Yasuhiro OHTA

Bilinearization and Casorati determinant solutions to non-autonomous $1+1$ dimensional discrete soliton equations

\section{MI2009-7 Yoshiyuki KAGEI}

Asymptotic behavior of solutions of the compressible Navier-Stokes equation around the plane Couette flow

MI2009-8 Shohei TATEISHI, Hidetoshi MATSUI \& Sadanori KONISHI

Nonlinear regression modeling via the lasso-type regularization

MI2009-9 Takeshi TAKAISHI \& Masato KIMURA

Phase field model for mode III crack growth in two dimensional elasticity

MI2009-10 Shingo SAITO

Generalisation of Mack's formula for claims reserving with arbitrary exponents for the variance assumption

MI2009-11 Kenji KAJIWARA, Masanobu KANEKO, Atsushi NOBE \& Teruhisa TSUDA Ultradiscretization of a solvable two-dimensional chaotic map associated with the Hesse cubic curve

\section{MI2009-12 Tetsu MASUDA}

Hypergeometric T -functions of the q-Painlevé system of type $E_{8}^{(1)}$

MI2009-13 Hidenao IWANE, Hitoshi YANAMI, Hirokazu ANAI \& Kazuhiro YOKOYAMA A Practical Implementation of a Symbolic-Numeric Cylindrical Algebraic Decomposition for Quantifier Elimination

MI2009-14 Yasunori MAEKAWA

On Gaussian decay estimates of solutions to some linear elliptic equations and its applications

MI2009-15 Yuya ISHIHARA \& Yoshiyuki KAGEI

Large time behavior of the semigroup on $L^{p}$ spaces associated with the linearized compressible Navier-Stokes equation in a cylindrical domain 
MI2009-16 Chikashi ARITA, Atsuo KUNIBA, Kazumitsu SAKAI \& Tsuyoshi SAWABE Spectrum in multi-species asymmetric simple exclusion process on a ring

MI2009-17 Masato WAKAYAMA \& Keitaro YAMAMOTO

Non-linear algebraic differential equations satisfied by certain family of elliptic functions

MI2009-18 Me Me NAING \& Yasuhide FUKUMOTO

Local Instability of an Elliptical Flow Subjected to a Coriolis Force

MI2009-19 Mitsunori KAYANO \& Sadanori KONISHI

Sparse functional principal component analysis via regularized basis expansions and its application

MI2009-20 Shuichi KAWANO \& Sadanori KONISHI

Semi-supervised logistic discrimination via regularized Gaussian basis expansions

MI2009-21 Hiroshi YOSHIDA, Yoshihiro MIWA \& Masanobu KANEKO

Elliptic curves and Fibonacci numbers arising from Lindenmayer system with symbolic computations

MI2009-22 Eiji ONODERA

A remark on the global existence of a third order dispersive flow into locally Hermitian symmetric spaces

MI2009-23 Stjepan LUGOMER \& Yasuhide FUKUMOTO

Generation of ribbons, helicoids and complex scherk surface in laser-matter Interactions

MI2009-24 Yu KAWAKAMI

Recent progress in value distribution of the hyperbolic Gauss map

MI2009-25 Takehiko KINOSHITA \& Mitsuhiro T. NAKAO

On very accurate enclosure of the optimal constant in the a priori error estimates for $H_{0}^{2}$-projection 
MI2009-26 Manabu YOSHIDA

Ramification of local fields and Fontaine's property (Pm)

MI2009-27 Yu KAWAKAMI

Value distribution of the hyperbolic Gauss maps for flat fronts in hyperbolic three-space

MI2009-28 Masahisa TABATA

Numerical simulation of fluid movement in an hourglass by an energy-stable finite element scheme

MI2009-29 Yoshiyuki KAGEI \& Yasunori MAEKAWA Asymptotic behaviors of solutions to evolution equations in the presence of translation and scaling invariance

MI2009-30 Yoshiyuki KAGEI \& Yasunori MAEKAWA

On asymptotic behaviors of solutions to parabolic systems modelling chemotaxis

MI2009-31 Masato WAKAYAMA \& Yoshinori YAMASAKI

Hecke's zeros and higher depth determinants

MI2009-32 Olivier PIRONNEAU \& Masahisa TABATA

Stability and convergence of a Galerkin-characteristics finite element scheme of lumped mass type

MI2009-33 Chikashi ARITA

Queueing process with excluded-volume effect

MI2009-34 Kenji KAJIWARA, Nobutaka NAKAZONO \& Teruhisa TSUDA

Projective reduction of the discrete Painlevé system of type $\left(A_{2}+A_{1}\right)^{(1)}$

MI2009-35 Yosuke MIZUYAMA, Takamasa SHINDE, Masahisa TABATA \& Daisuke TAGAMI Finite element computation for scattering problems of micro-hologram using DtN map 
MI2009-36 Reiichiro KAWAI \& Hiroki MASUDA

Exact simulation of finite variation tempered stable Ornstein-Uhlenbeck processes

MI2009-37 Hiroki MASUDA

On statistical aspects in calibrating a geometric skewed stable asset price model

MI2010-1 Hiroki MASUDA

Approximate self-weighted LAD estimation of discretely observed ergodic OrnsteinUhlenbeck processes

MI2010-2 Reiichiro KAWAI \& Hiroki MASUDA

Infinite variation tempered stable Ornstein-Uhlenbeck processes with discrete observations

MI2010-3 Kei HIROSE, Shuichi KAWANO, Daisuke MIIKE \& Sadanori KONISHI Hyper-parameter selection in Bayesian structural equation models

MI2010-4 Nobuyuki IKEDA \& Setsuo TANIGUCHI

The Itô-Nisio theorem, quadratic Wiener functionals, and 1-solitons

MI2010-5 Shohei TATEISHI \& Sadanori KONISHI

Nonlinear regression modeling and detecting change point via the relevance vector machine

MI2010-6 Shuichi KAWANO, Toshihiro MISUMI \& Sadanori KONISHI

Semi-supervised logistic discrimination via graph-based regularization

MI2010-7 Teruhisa TSUDA

UC hierarchy and monodromy preserving deformation

MI2010-8 Takahiro ITO

Abstract collision systems on groups

MI2010-9 Hiroshi YOSHIDA, Kinji KIMURA, Naoki YOSHIDA, Junko TANAKA \& Yoshihiro MIWA

An algebraic approach to underdetermined experiments 
MI2010-10 Kei HIROSE \& Sadanori KONISHI

Variable selection via the grouped weighted lasso for factor analysis models

MI2010-11 Katsusuke NABESHIMA \& Hiroshi YOSHIDA

Derivation of specific conditions with Comprehensive Groebner Systems

MI2010-12 Yoshiyuki KAGEI, Yu NAGAFUCHI \& Takeshi SUDOU

Decay estimates on solutions of the linearized compressible Navier-Stokes equation around a Poiseuille type flow

MI2010-13 Reiichiro KAWAI \& Hiroki MASUDA

On simulation of tempered stable random variates

MI2010-14 Yoshiyasu OZEKI

Non-existence of certain Galois representations with a uniform tame inertia weight

MI2010-15 Me Me NAING \& Yasuhide FUKUMOTO

Local Instability of a Rotating Flow Driven by Precession of Arbitrary Frequency

MI2010-16 Yu KAWAKAMI \& Daisuke NAKAJO

The value distribution of the Gauss map of improper affine spheres

MI2010-17 Kazunori YASUTAKE

On the classification of rank 2 almost Fano bundles on projective space

MI2010-18 Toshimitsu TAKAESU

Scaling limits for the system of semi-relativistic particles coupled to a scalar bose field

MI2010-19 Reiichiro KAWAI \& Hiroki MASUDA

Local asymptotic normality for normal inverse Gaussian Lévy processes with high-frequency sampling

MI2010-20 Yasuhide FUKUMOTO, Makoto HIROTA \& Youichi MIE

Lagrangian approach to weakly nonlinear stability of an elliptical flow 
MI2010-21 Hiroki MASUDA

Approximate quadratic estimating function for discretely observed Lévy driven SDEs with application to a noise normality test

MI2010-22 Toshimitsu TAKAESU

A Generalized Scaling Limit and its Application to the Semi-Relativistic Particles System Coupled to a Bose Field with Removing Ultraviolet Cutoffs

MI2010-23 Takahiro ITO, Mitsuhiko FUJIO, Shuichi INOKUCHI \& Yoshihiro MIZOGUCHI Composition, union and division of cellular automata on groups

MI2010-24 Toshimitsu TAKAESU

A Hardy's Uncertainty Principle Lemma in Weak Commutation Relations of Heisenberg-Lie Algebra

MI2010-25 Toshimitsu TAKAESU

On the Essential Self-Adjointness of Anti-Commutative Operators

MI2010-26 Reiichiro KAWAI \& Hiroki MASUDA

On the local asymptotic behavior of the likelihood function for Meixner Lévy processes under high-frequency sampling

MI2010-27 Chikashi ARITA \& Daichi YANAGISAWA

Exclusive Queueing Process with Discrete Time

MI2010-28 Jun-ichi INOGUCHI, Kenji KAJIWARA, Nozomu MATSUURA \& Yasuhiro OHTA

Motion and Bäcklund transformations of discrete plane curves

MI2010-29 Takanori YASUDA, Masaya YASUDA, Takeshi SHIMOYAMA \& Jun KOGURE On the Number of the Pairing-friendly Curves

MI2010-30 Chikashi ARITA \& Kohei MOTEGI

Spin-spin correlation functions of the $q$-VBS state of an integer spin model

MI2010-31 Shohei TATEISHI \& Sadanori KONISHI

Nonlinear regression modeling and spike detection via Gaussian basis expansions 
MI2010-32 Nobutaka NAKAZONO

Hypergeometric $\tau$ functions of the $q$-Painlevé systems of type $\left(A_{2}+A_{1}\right)^{(1)}$

MI2010-33 Yoshiyuki KAGEI

Global existence of solutions to the compressible Navier-Stokes equation around parallel flows

MI2010-34 Nobushige KUROKAWA, Masato WAKAYAMA \& Yoshinori YAMASAKI

Milnor-Selberg zeta functions and zeta regularizations

MI2010-35 Kissani PERERA \& Yoshihiro MIZOGUCHI

Laplacian energy of directed graphs and minimizing maximum outdegree algorithms

MI2010-36 Takanori YASUDA

CAP representations of inner forms of $S p(4)$ with respect to Klingen parabolic subgroup

MI2010-37 Chikashi ARITA \& Andreas SCHADSCHNEIDER

Dynamical analysis of the exclusive queueing process

MI2011-1 Yasuhide FUKUMOTO\& Alexander B. SAMOKHIN

Singular electromagnetic modes in an anisotropic medium

MI2011-2 Hiroki KONDO, Shingo SAITO \& Setsuo TANIGUCHI

Asymptotic tail dependence of the normal copula

MI2011-3 Takehiro HIROTSU, Hiroki KONDO, Shingo SAITO, Takuya SATO, Tatsushi TANAKA \& Setsuo TANIGUCHI

Anderson-Darling test and the Malliavin calculus

MI2011-4 Hiroshi INOUE, Shohei TATEISHI \& Sadanori KONISHI

Nonlinear regression modeling via Compressed Sensing

MI2011-5 Hiroshi INOUE

Implications in Compressed Sensing and the Restricted Isometry Property

MI2011-6 Daeju KIM \& Sadanori KONISHI

Predictive information criterion for nonlinear regression model based on basis expansion methods

MI2011-7 Shohei TATEISHI, Chiaki KINJYO \& Sadanori KONISHI

Group variable selection via relevance vector machine 
MI2011-8 Jan BREZINA \& Yoshiyuki KAGEI

Decay properties of solutions to the linearized compressible Navier-Stokes equation around time-periodic parallel flow

Group variable selection via relevance vector machine

MI2011-9 Chikashi ARITA, Arvind AYYER, Kirone MALLICK \& Sylvain PROLHAC Recursive structures in the multispecies TASEP

MI2011-10 Kazunori YASUTAKE

On projective space bundle with nef normalized tautological line bundle

MI2011-11 Hisashi ANDO, Mike HAY, Kenji KAJIWARA \& Tetsu MASUDA

An explicit formula for the discrete power function associated with circle patterns of Schramm type

MI2011-12 Yoshiyuki KAGEI

Asymptotic behavior of solutions to the compressible Navier-Stokes equation around a parallel flow

MI2011-13 Vladimír CHALUPECKÝ \& Adrian MUNTEAN

Semi-discrete finite difference multiscale scheme for a concrete corrosion model: approximation estimates and convergence

MI2011-14 Jun-ichi INOGUCHI, Kenji KAJIWARA, Nozomu MATSUURA \& Yasuhiro OHTA

Explicit solutions to the semi-discrete modified KdV equation and motion of discrete plane curves

MI2011-15 Hiroshi INOUE

A generalization of restricted isometry property and applications to compressed sensing

MI2011-16 Yu KAWAKAMI

A ramification theorem for the ratio of canonical forms of flat surfaces in hyperbolic three-space 